\title{
G

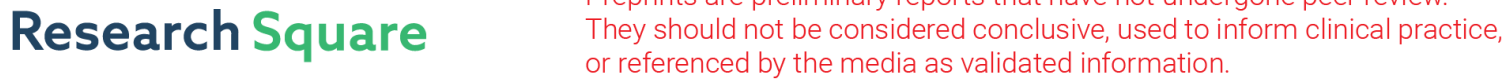 \\ Evolution of Pressure-induced Shear Amorphization in Rare-earth Hexaboride
}

\section{Rajamallu Karre}

Shanghai Jiao Tong University

\section{Yidi Shen}

University of Nevada-Reno

\section{Shuangxi Song}

Shanghai Jiao Tong University https://orcid.org/0000-0003-2425-8084

\section{Yixuan $\mathrm{Hu}$}

Shanghai Jiao Tong University

\section{Simanta Lahkar}

Shanghai Jiao Tong University https://orcid.org/0000-0002-6687-5227

\section{Xiaodong Wang}

Shanghai Jiao Tong University

\section{Bikramjit Basu}

Indian Institute of Science Bangalore

\section{Qi An}

University of Nevada Reno https://orcid.org/0000-0003-4838-6232

Kolan Reddy ( $\square$ kmreddy@sjtu.edu.cn )

Shanghai Jiao Tong University

\section{Article}

Keywords: rare-earth hexaborides, structural evolution, amorphous bands, nanoidentation, fragile hexaborides

Posted Date: October 5th, 2021

DOl: https://doi.org/10.21203/rs.3.rs-902567/v1

License: (c) (i) This work is licensed under a Creative Commons Attribution 4.0 International License. Read Full License

Version of Record: A version of this preprint was published at Communications Materials on April 22nd, 2022. See the published version at https://doi.org/10.1038/s43246-022-00246-2. 


\section{Abstract}

Research on rare-earth hexaborides mainly focuses on tuning the electronic structure from insulating-tometallic state and vice versa (referred as exotic phenomena) by high pressure experiments via displacive phase transformations, however, the structural evolution that contributing to this underlying failure mechanism remains not well understood. Herein, we examined the pressure induced structural evolution through a model system of europium hexaboride $\left(\mathrm{EuB}_{6}\right)$. Transmission electron microscopy reveal that the nanoscale amorphous shear bands mediated by dislocations play a decisive role in deformation failure of $\mathrm{EuB}_{6}$ subjected to high pressure nanoidentation at room temperature. Density functional theory simulations confirm that these amorphous bands evolve by breaking $B-B$ bonds within $\mathrm{B}_{6}$ octahedron of $\mathrm{EuB}_{6}$ during shear deformation. Our results underscore an important damage mechanism in hard and fragile hexaborides at high shear pressures.

\section{Introduction}

Rare-earth hexaborides $\left(\mathrm{RB}_{6}\right)$ have attracted considerable attention due to their extraordinary combination of electrical, magnetic, thermal and mechanical properties ${ }^{1}$. At ambient conditions, $\mathrm{RB}_{6}$ crystallizes in a cubic structure (i.e., CsCl-prototype, Space group: $\mathrm{Pm} m$ (no: 221)) ${ }^{2}$. The unique chemical bonding in $\mathrm{RB}_{6}$ arises from the octahedron $\mathrm{B}_{6}$ framework with large interstitial space to host a variety of the rare earth ions $(\mathrm{R}=\mathrm{Ba}, \mathrm{Ce}, \mathrm{Eu}, \mathrm{La}, \mathrm{Sm}, \mathrm{Sr}, \mathrm{Y}$ etc.) at the center of cubic crystal lattice. Despite having the same crystal structure, the properties of each hexaboride are uniquely different and diversified from each other due to their intrinsically strong correlated electron systems ${ }^{3}$. For instance, $\mathrm{CeB}_{6}$ shows dense Kondo effect and electric quadrupole ordering, $\mathrm{SmB}_{6}$ exhibits Kondo insulating and valence fluctuation, $\mathrm{LaB}_{6}$ have low work function for thermionic emission, $\mathrm{YB}_{6}$ possess remarkable superconductivity, $\mathrm{CaB}_{6}$ is semiconducting, and $\mathrm{EuB}_{6}$ displays ferromagnetic properties ${ }^{1,3}$.

Pressure-driven polymorphism has been predicted and observed in several hexaborides by first principle calculations and high-pressure X-ray experiments ${ }^{4-7}$. For instance, Kolmogorov et al. ${ }^{4}$ predicted that the thermodynamically unstable cubic ( ) $\mathrm{CaB}_{6}$ structure at high pressure compression transforms to an intermediate metastable orthorhombic oS28 structure around $13 \mathrm{GPa}$ before stabilizing to ground state tetragonal $t / 56$ structure above $32 \mathrm{GPa}$. Their experimental evidence showed that the structure in threedimensional morphology remains stable up to at least $28 \mathrm{GPa}$ but with certain distortion. Further, assistance of laser heating promoted the rebonding in strong covalent boron network above $31 \mathrm{GPa}$ and favored the ground state structure. Similarly, Zhu et al. ${ }^{5}$ examined a semiconducting $\mathrm{SrB}_{6}()$ and predicated new metallic polymorphs as orthorhombic $(\mathrm{Cmmm})$ and tetragonal $(14 / \mathrm{mmm})$ structures at high pressure of 48 and $60 \mathrm{GPa}$, respectively. These unique boron polyhedra are formed as a result of denser structural arrangement under high pressure and the formation enthalpies are decreased due to the additional B-B bonds of polyhedral networks ${ }^{5}$. In addition, they have also demonstrated a novel deformation mechanism with transient multicenter bonding yields in good combination of high strength 
and high ductility for the $14 / \mathrm{mmm}$ structure in $\mathrm{SrB}_{6}{ }^{5}$. These results indicate that upon high pressure produces the complex boron networks and structural transitions in hexaborides leading to unique properties, which has created immense interest for electromechanical systems ${ }^{3-7}$.

Here, we choose EuB $_{6}$ firstly because of its semi-metallic nature and its hardness (19-26 GPa) falling in range of $\mathrm{CaB}_{6}(16-28 \mathrm{GPa})$ and $\mathrm{SrB}_{6}(18.5-27 \mathrm{GPa})^{8}$. Secondly, $\mathrm{EuB}_{6}$ is the only hexaboride with ferromagnetic order and low thermal expansion coefficient, which can be tuned to ground state electron system. Thirdly, single phase $\mathrm{EuB}_{6}$ in bulk material can be easily produced using various sintering methods ${ }^{2,9,10}$. In addition to these applications, $\mathrm{EuB}_{6}$ received significant attention in hexaboride family as a potential second phase material for structural applications owing to its high thermal stability and high neutron absorption properties ${ }^{9,11}$.

In this study, we used nanoindentation to impose high pressures on $\mathrm{EuB}_{6}$ crystals, followed by microstructural characterization using Raman spectroscopy and spherical aberration (Cs) corrected TEM. We found beneath the indentation surface, the nanoscale amorphous shear bands preceded by the emission and propagation of dislocations. The DFT simulations, which agree with experiment, reveals that the chemical bond breaking within octahedral $B_{6}$ initiates the mechanical instability leading to the localized amorphization in $\mathrm{EuB}_{6}$ by high pressure shear deformation.

\section{Methods}

Material fabrication and nanoindentation experiments. The composite $\mathrm{B}_{4} \mathrm{C}$-EuB 6 samples were fabricated using spark plasma sintering (SPS) technique with precursors of $\mathrm{Eu}_{2} \mathrm{O}_{3}\left(\mathrm{~d}_{50} \sim 0.7 \mu \mathrm{m}, 99 \%\right.$ purity) and $\mathrm{B}_{4} \mathrm{C}\left(\mathrm{d}_{50} \sim 5 \mu \mathrm{m}, 99 \%\right.$ purity) powders. The powders were wet-milled for 2 hours with $150 \mathrm{rpm}$ using planetary ball mill (Fritsch, Germany). Then this ball milled powder was dried in a vacuum oven at $80^{\circ} \mathrm{C}$ for 3 hours. Finally, samples were loaded in an electrically conducting die and consolidated by SPS (Dr. Sinter, Japan) using sintering temperature of $1900{ }^{\circ} \mathrm{C}$, held for $15 \mathrm{~min}$ with the applied pressure of 45 $\mathrm{MPa}$. The rectangular specimens were cut from the bulk sintered $\mathrm{B}_{4} \mathrm{C}-\mathrm{EuB}_{6}$ composite using slow speed diamond cutter and polished with diamond film sheets i.e., 30, 15, 9, 6 and $1 \mu \mathrm{m}$ for microstructural characterization and nanoindentation tests. Nanoindentation (Hysitron T1950) in load control mode was utilized for determining the mechanical properties of $\mathrm{EuB}_{6}$. In this regard, diamond Berkovich tip was used with maximum load of $10 \mathrm{mN}$ held for $2 \mathrm{sec}$. The loading and unloading rate were kept $1 \mathrm{mN} / \mathrm{s}$. Also, a series of indentations at a maximum applied load of $500 \mathrm{mN}$ were performed on EuB ${ }_{6}$ crystals with MTS G200 system equipped with a Berkovich indenter.

Microstructural characterization. The XRD of as-synthesized samples was obtained by using Ultima IV (Rigaku, Japan) with Cu Ka $(\lambda=1.5418 \AA)$, operated at $40 \mathrm{kV}$ and $30 \mathrm{~mA}$. The microstructural analysis of EuB $_{6}$ was carried out using SEM (Nova NanoSEM 450 (FEI)). Further, the electron backscattered diffraction (EBSD) patterns were acquired to obtain crystallographic orientation of $\mathrm{EuB}_{6}$ and SEM-EDS 
was used for elemental mapping analysis. Raman micro spectroscope was carried out using Renishaw inVia Qontor with an excitation wavelength of $532 \mathrm{~nm}$ that has laser beam spot $1 \mu \mathrm{m}$. The crosssectional TEM specimens underneath the indentation were prepared by standard lift-out technique using focused ion beam (FIB) (VERSA 3D (FEI, USA)) method. The aberration-corrected TEM (JEM-ARM 200F (JEOL, Japan)) operated at $200 \mathrm{kV}$ was utilized for obtaining high resolution atomic images. Further, STEM-EDS was performed for chemical analysis. The atomic TEM images were simulated using the software package of HREM Research Inc., (Japan). Local strain measurements from HRTEM images of deformation regions have been analysis using peak pair analysis software ${ }^{32}$.

Computational methodology. The DFT calculations were performed using Vienna ab initio simulation package (VASP) with plane wave basis set ${ }^{33,34}$. The generalized gradient approximation (GGA) type Perdew-Burke-Ernzerhof (PBE) exchange-correlation functional were implemented for electronic exchange and correlation interaction ${ }^{35,36}$. The pseudopotentials of $B$ and Eu were generated using the projector augmented wave (PAW) method with $2 s^{2} 2 p^{1}$ and $5 p^{6} 6 s^{2}$ treated as valence electrons ${ }^{36}$. The energy cutoff of $500 \mathrm{eV}$ was set in all simulations for the good convergence of energy, force, stress and geometries. The energy convergence of $10^{-6} \mathrm{eV}$ for terminating electronic self-consistent field (SCF) and the force criterion $10^{-3} \mathrm{eV} / \AA \AA$ were used in all simulations. $\mathrm{A} \Gamma$-centered k-point mesh method was used for Brillouin zone integration with the mesh density a high resolution above $2 \pi \times 1 / 40 \AA^{-1}$.

For the pure shear and biaxial shear deformations of $\mathrm{EuB}_{6}$, we considered (2 2)[120] and (110)[1 0] as plausible slip systems under both pure and biaxial shear deformation. For shear along (2 2)[120] slip system we used a supercell model which contains 63 atoms while we used a 56-atom supercell for (110) [1 0 ] slip system. The $(4 \times 7 \times 3)$ and $(3 \times 9 \times 3)$ K-point meshes were used for $(22)[120]$ and $(110)[10]$ shear deformations, respectively. $\mathrm{EuB}_{6}$ is one of hexaboride compounds and belongs to the space group of $\mathrm{Pm} \mathrm{m}$ in which the unit cell contains one $\mathrm{B}_{6}$ octahedron and one Eu atom ${ }^{2}$. In each $\mathrm{B}_{6}$ octahedron, $6 \mathrm{~B}$ atoms are covalently bonded to 6 nearby octahedra. Thus, based on Wade's rules ${ }^{37}$, each $B_{6}$ octahedron requires 14 electrons to stabilize the close octahedral structure. In addition, 6 B-B bonds connecting two nearby octahedra also need extra 6 electrons for each octahedron. However, $6 \mathrm{~B}$ atoms in each octahedron can only provide 18 electrons. Thus, the Eu atoms are required to transfer 2 electrons to octahedron to satisfy the Wade's rules ${ }^{37}$.

\section{Results}

Structure characterization of as-synthesized $\mathrm{EuB}_{6}$. The microstructure of as-synthesized EuB -boron $_{6}$ carbide $\left(\mathrm{B}_{4} \mathrm{C}\right)$ composite is shown in Fig. 1. In Fig. 1(a) scanning electron microscopy (SEM) image shows the bright grains having faceted cuboidal corresponding to $\mathrm{EuB}_{6}$ crystals which are surrounded by the grey region of $\mathrm{B}_{4} \mathrm{C}$ matrix. Fig. $1(\mathrm{~b})$ electron back scattered diffraction (EBSD) pattern of $\mathrm{EuB}_{6}$ acquired from the same region of Fig. 1(a), displays the crystallographic orientation for the grain facets of square as (100) plane, rectangle as (110) plane and triangle as (111) plane, respectively. High 
resolution TEM (HRTEM) images of $\mathrm{EuB}_{6}$ are projected along the [100] and [120] zone axes in Fig. 1(c) and 1 (d) as confirmed by selected area electron diffraction (SAED) patterns shown in the inset at the upper right corner of Fig. 1(c) and (d). Further, simulated atomic images projected along the same zone axes are superimposed at the lower right corner of the HRTEM images (Fig. 1(c) and 1(d)). The comparison of experimental and simulated TEM images (Fig. 1(c) and 1(d)) reveals that the bright spots belong to europium $(\mathrm{Eu})$ individual atomic sites and grey spots are corresponding to the boron $\left(\mathrm{B}_{6}\right)$ clusters, where Eu and B atoms are represented by open circles with magenta and green colors, respectively.

Raman Spectroscopy of pristine and deformed $\mathrm{EuB}_{6}$. In order to determine the mechanical properties and introduce structural transformations in $\mathrm{EuB}_{6}$ crystals, we performed nanoindentation on different facets of $\mathrm{EuB}_{6}$. The measured hardness and elastic modulus for (100), (110) and (111) facets are $28.52 \pm 0.42$ $\mathrm{GPa}, 28.88 \pm 0.72 \mathrm{GPa}, 27.72 \pm 1.05 \mathrm{GPa}$ and $231 \pm 2.5 \mathrm{GPa}, 240 \pm 5 \mathrm{GPa}, 225 \pm 8.7 \mathrm{GPa}$, respectively. Fig. 2(a) shows the representative SEM image of residual indentation taken on the grain of square ((001) plane) facet. The Raman spectra acquired from the residual indent (black solid line) and pristine (red dotted line) of $\mathrm{EuB}_{6}$ are presented in Fig. 2(b). Since $\mathrm{EuB}_{6}$ belongs to the cubic $\mathrm{O}_{\mathrm{h}}$ group, there are three Raman active $\left(T_{2 g}, E_{g}\right.$ and $\left.A_{1 g}\right)$ phonons caused by internal displacement of boron atoms in the octahedron $\mathrm{B}_{6}{ }^{12}$. In this study, these characteristic peaks are found at $768 \mathrm{~cm}^{-1}, 1112 \mathrm{~cm}^{-1}$ and $1256 \mathrm{~cm}^{-}$ ${ }^{1}$, respectively, which are in good agreement with the previous reports ${ }^{6,10}$. All the Raman peaks in Fig. 2(b) are related to the vibrations of octahedral $B_{6}$. The $E_{g}$ mode is associated with compressing up and down vibrations, while $T_{2 g}$ mode is related to the scissoring displacement of $B$ atoms in the octahedral $B_{6}{ }^{13}$. Therefore, both $T_{2 g}$ and $E_{g}$ modes are modulating the B-B-B bond angles, whereas, $A_{1 g}$ mode is related to the stretching of $B-B$ bond ${ }^{13}$. From residual indent (Fig. 2(b)), peak broadening of $T_{2 g}$ and $E_{g}$ peaks is apparent suggesting that the structural transformations in $\mathrm{EuB}_{6}$ may have occurred in B-B-B bonds of octahedra $\mathrm{B}_{6}$ sites upon indentation induced high pressures.

Structural evolution observations of indentation-induced $\mathrm{EuB}_{6}$. In order to elucidate the underlying mechanism that drives the structural transformations in $\mathrm{EuB}_{6}$ during high pressure deformation, crosssectional TEM specimens from multiple crystallographic orientations were prepared from the residual indents using focused ion beam (FIB) milling ${ }^{14,15}$ (Fig. 3 and Supplementary text and Supplementary Fig. S1). Fig. 3(a) shows low magnification TEM image of indentation impression on the (001) surface of $\mathrm{EuB}_{6}$ cuboidal square. The microcracks and nanoscale bands (marked with dark and white arrow heads, respectively in Fig. 3(a)) can be seen from low TEM magnification image, indicating severe damage and plastic deformation within the indentation region. These observed bands have width of $\sim 3$ to $8 \mathrm{~nm}$ and length ranging from 100 to $500 \mathrm{~nm}$. The magnified (Fig. 3(b)) TEM image shows a single band length of about $120 \mathrm{~nm}$, which is surrounded by high density of planar defects (indicated by red arrows). HRTEM image acquired from the nanosized band reveal the loss of crystallinity (Fig. 3(c)). Fast Fourier transforms (FFT) taken from the region $\mathrm{B}$, shows a diffuse halo without any diffraction spots, confirming the occurrence of amorphous structure within the band. On the other hand, FFT pattern taken from the 
crystalline structure on either side of band along the [120] crystallographic direction displays Pm $m$ symmetry of $\mathrm{EuB}_{6}$ (Inset A). Further, FFTs confirm the formation of amorphous band roughly aligned on a (2 2) plane. The measured angle between the (001) surface plane and slip plane is about $48^{\circ}$ suggesting that the amorphous bands are induced by high shear stresses. Fig. 3(d) is the HRTEM image taken from tip of the amorphous band, as shown in white box of Fig. 3(a). The Burgers loop from the tip of amorphous band drawn by white dotted lines in Fig. 3(d) reveals obvious shear displacement of about $\sim 2 \AA$. Moreover, Eu and B atomic overlay in Fig. 3(d) at near amorphous band providing evidence of crystal lattice deregister from the boron clusters, rather than Eu atoms. Fig. 3(e) is the grid generated through the Peak Pair Analysis algorithm in the same area. The grid lines in image (Fig. 3(e)) clearly shows significant lattice bending before the initiation of dislocations at the tip of band. Analysis of shear strain $\left(e_{x y}\right)$ map from the area (Fig. 3(e)), specifically identifies a maximum absolute shear strain of about $25 \%$ (Fig. 3(f)) as estimated from the proximity of dislocation core in a nano-strained band region compared to the parent crystalline structure. We also characterized the indentation impression profuse on the (011) rectangle surface of $\mathrm{EuB}_{6}$ (Supplementary Fig. S2). TEM characterization along the zone axis (Supplementary Fig. S2 $(a, b)$ ) shows the nanosized amorphous bands roughly aligned parallel to the plane. A HRTEM image obtained from the tip of amorphous band identifies the dislocation core (Supplementary Fig. S2(c)). These observations indicate that these dislocations are geometrically necessary to mediate the amorphization process in $\mathrm{EuB}_{6}$.

\section{DFT prediction of shear amorphization in $\mathrm{EuB}_{6}$}

Based on the TEM observations (Fig.3 and Fig. S2), we assumed that the (2 2)[120] and (110)[1 0] are two possible slip systems to initiate the deconstruction of $B_{6}$ octahedra and formation of amorphous bands in $\mathrm{EuB}_{6}$. Hence, we applied pure shear deformation on $\mathrm{EuB}_{6}$ along these two slip systems. The obtained shear-stress-shear-strain relationship is shown in Fig. 4(a). The ideal shear strength along (2 2 ) [120] is $29.09 \mathrm{GPa}$, which is much lower than that (41.67 GPa) along (110)[1 0]. This suggests that (2 2) [120] is more plausible slip system, which agrees very well with the experimental observation. Then, we examined the deformation and failure mechanism of $\mathrm{EuB}_{6}$ along (2 2)[120] slip system, as shown in Fig. 4(b-f). The intact structure before shear is shown in Fig. 4(b). It is worth noting that the structure is shown along " $\mathrm{B}$ " axis ([ 01] direction) rather than " $\mathrm{A}$ " axis (shear direction of [120]) to better describe the failure mechanism. The atomic structure viewed along [120] direction is shown in Supplementary Fig. S3. As shear strain increases to 0.465 , the B22-B32 bond between two octahedra is gradually stretched from original $1.68 \AA$ to $2.15 \AA$ without breaking, as shown in Fig. 4(c). When the shear strain continuously increases to 0.489 , the B22-B32 bond is stretched to $2.26 \AA$ and breaks, as shown in Fig. 4(d). This bond breaking also slightly releases the shear stress from the maximum of $29.09 \mathrm{GPa}$ to $28.29 \mathrm{GPa}$. Then, with the increase of shear strain, B22 and B32 atoms move far from each other, leading to further decrease of shear stress. In particular, at 0.514 shear strain, the distance between B22 and B32 atoms is $2.42 \AA$, as shown in Fig. 4(e). Finally, at 0.539 shear strain, the B16-B28 bond in the octahedron is stretched from original $1.75 \AA$ to $1.90 \AA$ and breaks (Fig. 4(f)), initiating the deconstruction of octahedra, which agrees with the results in experiments. This structural failure further releases the shear stress to $5.33 \mathrm{GPa}$. 
In addition to (2 2)[120], we also examined the failure mechanism of EuB 6 along (110)[1 0], as shown in Supplementary Fig. S4. Supplementary Fig. S4(a) shows the intact structure. At 0.245 shear strain corresponding to the ideal shear strength, the B25-B32 connecting two nearby octahedra is gradually stretched from original $1.68 \AA$ to $2.13 \AA$, as shown in Supplementary Fig. S3(b). However, this bond is not broken. Then, at 0.263 shear strain, the B25-B32 bond is drastically stretched to $2.36 \AA$ and breaks, as shown in Fig. S4(c). This bond breaking releases shear stress to $39.65 \mathrm{GPa}$. After that, with increase of shear strain, the B25 and B32 atoms is stretched far from each other, leading to the further release of shear stress. Particularly, at 0.297 shear strain, the distance of B25 and B32 atoms is $2.88 \AA$, as shown in Fig. S4(d). The corresponded shear stress also decreases to $27.01 \mathrm{GPa}$. The above results indicate that the failure mechanism of $\mathrm{EuB}_{6}$ under ideal shear deformation arises from the breaking of $\mathrm{B}-\mathrm{B}$ bonds that connect two nearby octahedra first and then the deconstruction of octahedra.

To mimic the complex stress condition in the indentation experiments, we also applied biaxial shear deformation along the plausible slip system of (22)[120]. The shear-stress-shear-strain relationship of $\mathrm{EuB}_{6}$ along (2 2)[120] is shown for biaxial shear deformation in Fig. 5. The ideal shear strength is 23.31 GPa (Fig. 5(a)), which is lower than 29.09 GPa for ideal shear deformation (Fig. 4(a)). The obtained failure mechanism is shown in Fig. 5(b-f). The initial structure is shown in Fig. 5(b). At 0.209 shear strain, the shear stress reaches to its maximum value of 23.31GPa. The B35-B16 and B53-B23 bonds in octahedron are slightly stretched from original $1.75 \AA$ to $1.78 \AA$ and $1.82 \AA$, respectively, as shown in Fig. 5 (c). Then, when the shear strain continuously increases to 0.232 , these bonds are further stretched to

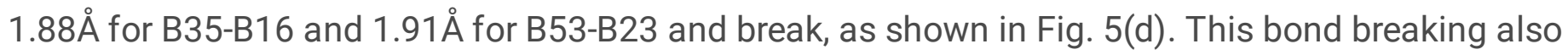
releases the stress from $23.31 \mathrm{GPa}$ to $15.71 \mathrm{GPa}$. As shear strain further increases to 0.276 , the B53-B6 bond is gradually stretched to $1.92 \AA$, as shown in Fig. 5(e). However, it does not break at this shear strain. Next, at 0.299 shear strain, the B53 atom is stretched out of octahedron and B6-B53 bond breaks, as shown in Fig. 5(f). The deconstruction of octahedron releases the shear stress to $4.96 \mathrm{GPa}$. Therefore, the failure mechanism of $\mathrm{EuB}_{6}$ under biaxial shear deformation arises from the $\mathrm{B}-\mathrm{B}$ bond breaking within the octahedra. This deconstruction of octahedra will initiate the formation of amorphous shear in $\mathrm{EuB}_{6}$, which further verifies the observation in the experiments.

\section{Discussion}

Amorphization has been observed in a wide variety of hard and complex crystal structures including diamond ${ }^{16}$, ice ${ }^{17}$, minerals ${ }^{18}$, semiconductors $(\mathrm{Si}, \mathrm{Ge}, \mathrm{GaAs}, \mathrm{InSb})^{19-21}$, ceramics $\left(\mathrm{Al}_{2} \mathrm{O}_{3}, \mathrm{SiC}, \mathrm{B}_{4} \mathrm{C}, \mathrm{B}_{6} \mathrm{O}\right.$, $\left.\mathrm{Si}_{3} \mathrm{~N}_{4}\right)^{22-24}$ and intermetallics $\left(\mathrm{TiNi}_{1} \mathrm{Ni}_{3} \mathrm{Al}, \mathrm{SmCO}_{5}\right)^{25}$ and are generally related to the pressure-induce lattice destabilization ${ }^{15-25}$. Similarly, hexaborides possess strong directional bonding with relatively open structure. Thus, conventional plastic deformation associated with motion of crystal defects is very difficult at room temperature. Therefore, hexaborides crystalline structures are expected to fail easily on the relative weaker planes to became denser phase under high pressures. Additionally, structural transformations or local softening in the covalent or ionic solid materials are vastly dependent on type of applied pressure conditions. Previous studies on $\mathrm{CaB}_{6}$ and $\mathrm{SrB}_{6}$ hexaborides using diamond anvil cell 
(DAC) experiments under quasi-hydrostatic media demonstrated the polymorphic transition from a simple cubic phase into complex and more denser boron network orthorhombic and tetragonal phases ${ }^{4,5}$, with the assistance of temperature (laser-heating) to produce irreversible bonding for inducing structural transformations. In another study on $\mathrm{EuB}_{6}$ under hydrostatic high pressures above $20 \mathrm{GPa}$ showed magnetic transitions from ferromagnetic to paramagnetic phase with mixed valence states ${ }^{26}$. Further, temperature dependent Raman scattering and first-principle calculations showed evidence that the cubic symmetry breaking induces a non-cubic environment in the $\mathrm{B}_{6}$ octahedron in $\mathrm{EuB}_{6}{ }^{6}$. All these studies performed by hydrostatic or quasi-hydrostatic DAC experiments have not predicted or reported amorphization in hexaborides ${ }^{4-6,26}$. However, a study by Yan et al. ${ }^{27}$ reported amorphization in superhard $\mathrm{B}_{4} \mathrm{C}$ during depressurization in a non-hydrostatic stress using $\mathrm{DAC}$, while in hydrostatic or quasihydrostatic stresses the amorphization was not detected suggesting the nature of the applied stresses play an important role in mechanical instabilities of crystalline structure.

Here we used nanoindentation to induce structural transformations in $\mathrm{EuB}_{6}$, which comprises both hydrostatic and non-hydrostatic (shear) stresses. Our TEM observations beneath the indent surface showed that the nano-sized amorphous bands are initiated by dislocations. Such nucleation of dislocations is believed to evolve within the indentation region of a material as the applied load approaches above the critical resolve shear stress (CRSS) ${ }^{28-30}$. The measured angle between indentation surface plane and amorphous slip plane of $\mathrm{EuB}_{6}$ is approximately $40-50^{\circ}$ suggesting shear stresses play vital role to this process (Fig. 3). The DFT simulations further provided evidence of crystal-to-amorphous transition along two major slip systems of $\mathrm{EuB}_{6}$. Under pure-shear deformation ((Fig. 4 and Fig. S4)), the B-B bonds in the octahedron is gradually stretched and finally the structure failed initiating the deconstruction of octahedra. While in the biaxial shear deformation ${ }^{31}$ (Fig. 5), the failure is caused directly by breaking of B-B bond within the octahedron of $\mathrm{EuB}_{6}$ (Fig. 4). This result of biaxial shear is consistent with the bond distortions beneath the residual indent observed by micro-Raman spectroscopy (Fig. 2). Therefore, our study confirms that the nanoscale amorphous bands in $\mathrm{EuB}_{6}$ is mediated by the dislocations on relatively easier slip planes by breaking the B-B bond of octahedral $\mathrm{B}_{6}$ during the shear deformation.

In summary, we employed the nanoindentation to investigate the structural transitions on a $\mathrm{EuB}_{6}$, combining with Raman spectroscopy, Cs-corrected TEM and DFT simulations. We found localized amorphization in $\mathrm{EuB}_{6}$ is mediated by dislocations on very specific crystallographic orientations. The simulations indicate that this amorphization process is triggered by breaking B-B chemical bonds in octahedral $\mathrm{B}_{6}$ upon shear deformation, in line with experimental observations. We believe that our observation underlying mechanism of shear induced amorphization mediated by dislocations in a model hexaboride system offers opportunities for controlling the exotic states deformation structures and to tune their properties at high pressures.

\section{Declarations}




\section{Authors Contributions}

K.M.R. conceived and supervised this study. K.M.R, R.K, Y.H. and X.W. did microstrostructural characterization. S. S. prepared FIB cross-sectioned specimens. Y.S and Q.A. conducted the DFT simulations. S. L. contributed to Strain mapping analysis. B. B. coordinated the specimen. R.K, Y.S, Q.A and K.M.R wrote the manuscript. All the authors discussed the results and commented on the manuscript.

\section{Declaration of Competing Interest}

The authors declare that they have no competing interests.

\section{Acknowledgements}

The work is financially supported by National Science Foundation China (Grant No. 51850410501) and seed funding from the School of Materials Science and Engineering at Shanghai Jiao Tong University (China).

\section{Additional Information}

Supplementary information is provided along with this manuscript

\section{References}

1. Cahill, J. T. \& Graeve, O. A. Hexaborides: A review of structure, synthesis and processing. J. Mater. Res. Technol. 8, 6321-6335 (2019).

2. Futamoto, M., Aita, T. \& Kawabe, U. Microhardness of hexaboride single crystals. Mater. Res. Bull. 14, 1329-1334 (1979).

3. Sun, L. \& Wu, Q. Pressure-induced exotic states in rare earth hexaborides. Reports Prog. Phys. 79, 084503 (2016).

4. Kolmogorov, A. N., Shah, S., Margine, E. R., Kleppe, A. K. \& Jephcoat, A. P. Pressure-driven evolution of the covalent network in $\mathrm{CaB}_{6}$. Phys. Rev. Lett. 109, 075501 (2012).

5. Zhu, L., Borstad, G. M., Cohen, R. E. \& Strobel, T. A. Pressure-induced polymorphism in $\mathrm{SrB}_{6}$ and deformation mechanisms of covalent networks. Phys. Rev. B 100, 214102 (2019).

6. Martinho, $\mathrm{H}$. et al. Evidence for a subtle structural symmetry breaking in $\mathrm{EuB}_{6}$. J. Phys. Condens. Matter 21, 456007 (2009).

7. Duan, L. et al. Pressure induced semiconductor-semimetal-superconductor transition of magnesium hexaborides. Dalt. Trans. 48, 14299-14305 (2019).

8. Zhou, Y., Dai, F., Xiang, H., Liu, B. \& Feng, Z. Shear anisotropy: Tuning high temperature metal hexaborides from soft to extremely hard. J. Mater. Sci. Technol. 33, 1371-1377 (2017). 
9. Sonber, J. K., Murthy, T. S. R. C., Subramanian, C., Hubli, R. C. \& Suri, A. K. Synthesis, densification and characterization of $\mathrm{EuB}_{6}$. Int. J. Refract. Met. Hard Mater. 38, 67-72 (2013).

10. Selvan, R. K., Genish, I., Perelshtein, I., Calderon Moreno, J. M. \& Gedanken, A. Single step, lowtemperature synthesis of submicron-sized rare earth hexaborides. J. Phys. Chem. C112, 1795-1802 (2008).

11. Wu, P. C. S. Europia as a nuclear control material. Nucl. Technol. 39, 84-94 (1978).

12. Udagawa, M. et al. Raman scattering investigation of high temperature ferromagnetic crystals. J. Phys. Soc. Japan 71, 314-316 (2002).

13. Nguyen, T. H. et al. Raman spectroscopic evidence of impurity-induced structural distortion in $\mathrm{SmB}_{6}$. J. Raman Spectrosc. 50, 1661-1671 (2019).

14. Giannuzzi, L. A. \& Stevie, F. A. A review of focused ion beam milling techniques for TEM specimen preparation. Micron 30, 197-204 (1999).

15. Reddy, K. M., Liu, P., Hirata, A., Fujita, T. \& Chen, M. W. Atomic structure of amorphous shear bands in boron carbide. Nat. Commun. 4, 2483 (2013).

16. Pastweka, L., Moser S., Gumbsch P. \& Moseler M. Anisotropic mechanical amorphization drives wear in diamond. Nat. Mater. 10, 34-38 (2011).

17. Mishima. O., Calvert L. D. \& Whalley, E. 'Melting ice' I at $77 \mathrm{~K}$ and 10 kbar: a new method of making amorphous solids. Nature 310, 393-395 (1984).

18. Samae, V. et al. Stress-induced amorphization triggers deformation in the lithospheric mantle. Nature 591, 82-86 (2021).

19. Clarke, D. R., Kroll, M. C., Kirchner, P. D. \& Cook, R. F. Amorphization and conductivity of silicon and germanium induced by indentation. Phys. Rev. Lett. 60, 2156-2159 (1988).

20. Kailer, A., Nickel, K. G. \& Gogotsi, Y. G. Raman microspectroscopy of nanocrystalline and amorphous phases in hardness indentations. J. Raman Spectrosc. 30, 939-946 (1999).

21. Zhao. S. et al. Directional amorphization of covalently-bonded solids: A generalized deformation mechanism in extreme loading. Mater. Today (2021).

22. Schmücker, M., Schneider, H. \& Kriven, W. M. Indentation-induced amorphization in mullite single crystals. J. Am. Ceram. Soc. 86, 1821-1822 (2003).

23. Chen, M. W., McCauley, J. W. \& Hemker, K. J. Shock-Induced localized amorphization in boron carbide. Science 299, 1563-1566 (2003).

24. Madhav Reddy, K. et al. Shear amorphization of boron suboxide. Scr. Mater. 76, 9-12 (2014).

25. Karre, R. et al. Vacancy-driven shear localization in silicon nitride. Scr. Mater. 190, 163-167 (2021).

26. Luo, H. et al. Plasticity without dislocations in a polycrystalline intermetallic. Nat. Comm. 10, 3587 (2019).

27. Kutelak, L. O. High pressures magnetism of EuB 6 probed with X-ray spectroscopy techniques. (2020). 
28. Yan, X. Q. et al. Depressurization amorphization of single-crystal boron carbide. Phys. Rev. Lett. 102, 075505 (2009).

29. Schuh, C. A. Nanoindentation studies of materials. Mater. Today 9, 32-40 (2006).

30. Jang, J. II, Lance, M. J., Wen, S., Tsui, T. Y. \& Pharr, G. M. Indentation-induced phase transformations in silicon: Influences of load, rate and indenter angle on the transformation behavior. Acta Mater. 53, 1759-1770 (2005).

31. Reddy, K. M. et al. Dislocation-mediated shear amorphization in boron carbide. Sci. Adv. 7, eabc6714 (2021).

32. Li, B., Sun, H. \& Chen, C. Large indentation strain-stiffening in nanotwinned cubic boron nitride. Nat. Commun. 5, 4965 (2014).

33. Galindo, P. L. et al. The peak pairs algorithm for strain mapping from HRTEM images. Ultra Microscopy. 107, 1186-1193 (2007)

34. Kresse, G. \& Furthmüller, J. Efficiency of ab-initio total energy calculations for metals and semiconductors using a plane-wave basis set. Comput. Mater. Sci. 6, 15-50 (1996).

35. Kresse, G. \& Furthmüller, J. Efficient iterative schemes for ab initio total-energy calculations using a plane-wave basis set. Phys. Rev. B 54, 11169 (1996).

36. Kresse, G. \& Hafner, J. Ab initio molecular dynamics for liquid metals. Phys. Rev. B 47, 558-561 (1993).

37. Kresse, G. \& Joubert, D. From ultrasoft pseudopotentials to the projector augmented-wave method. Phys. Rev. B - Condens. Matter Mater. Phys. 59, 1758-1775 (1999).

38. Welch, A. J. The significance and impact of Wade's rules. Chem. Commun. 49, 3615 (2013).

\section{Figures}



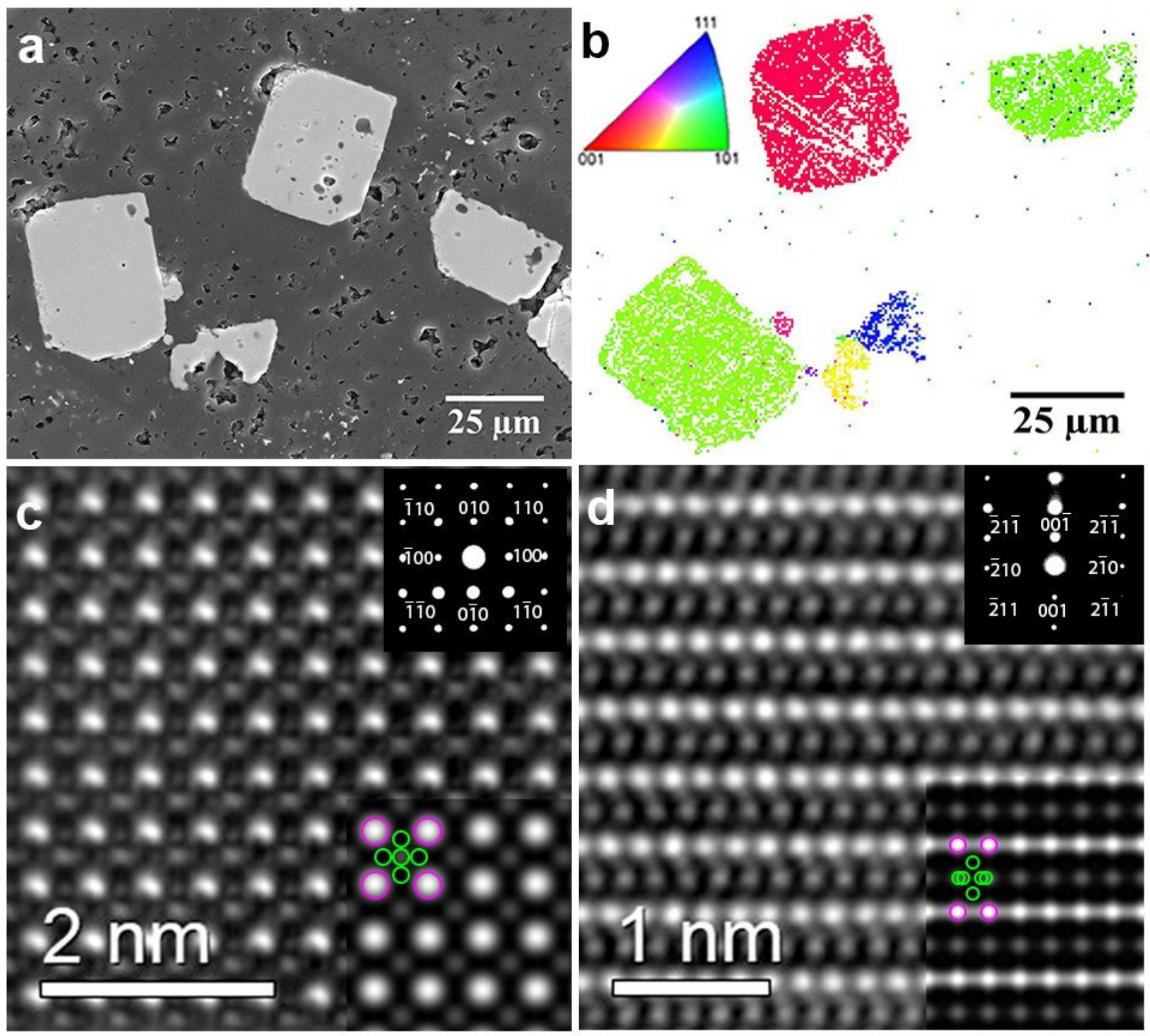

\section{Figure 1}

Electron microscopy characterization of as-synthesized EuB6. a Secondary electron SEM image shows microstructure of EuB6-B4C composite. b Corresponding EBSD pattern of EuB6 phase. $c$ and d High resolution TEM images of EuB6 along [100] and [120] zone axes, respectively. Simulated HRTEM images are superimposed on the rightmost lower corner of the images. The boron (B) and europium (Eu) atoms are represented by open circles with green and magenta colors, respectively. The indexed DP's along same zone axes are also shown at the rightmost upper corner of the images. 

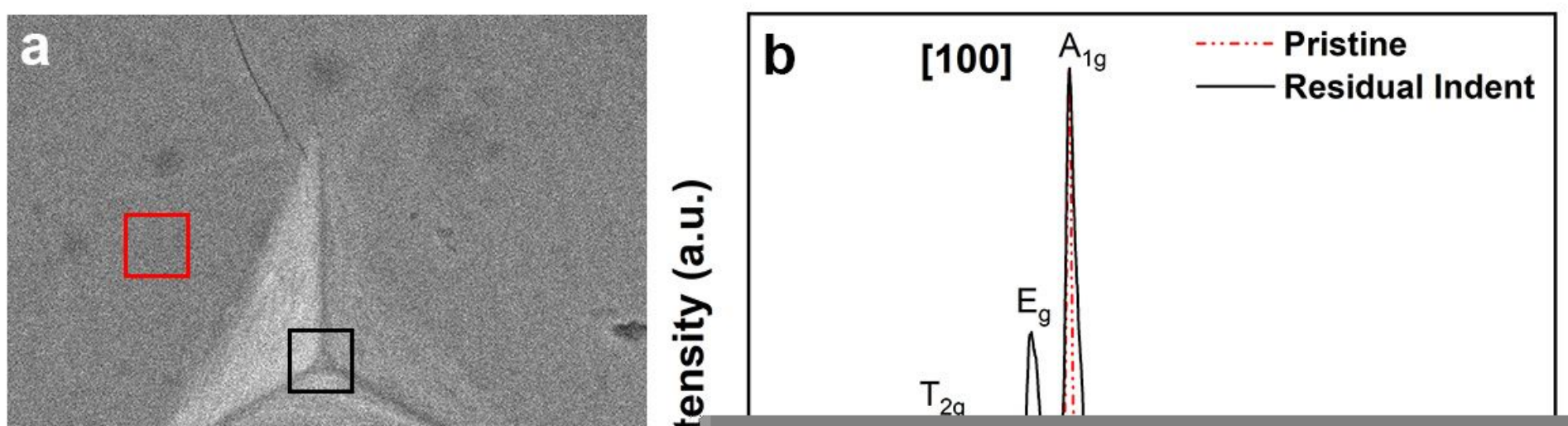

Figure 2

Raman spectroscopy studies of EuB6. a SEM image shows Berkovich-indented impression at applied of $500 \mathrm{mN}$ on square cuboid ([100] orientation) of EuB6. Here, the square boxes illustrate the regions from which the micro-Raman spectra acquired; black and red boxes represent the residual indent and pristine regions, respectively. b Raman spectra of EuB6 from residual indent (black line) and pristine (red-dotted line) regions show three major peaks T2g (768 cm-1), Eg (1112 cm-1) and A2g (1256 cm-1). 

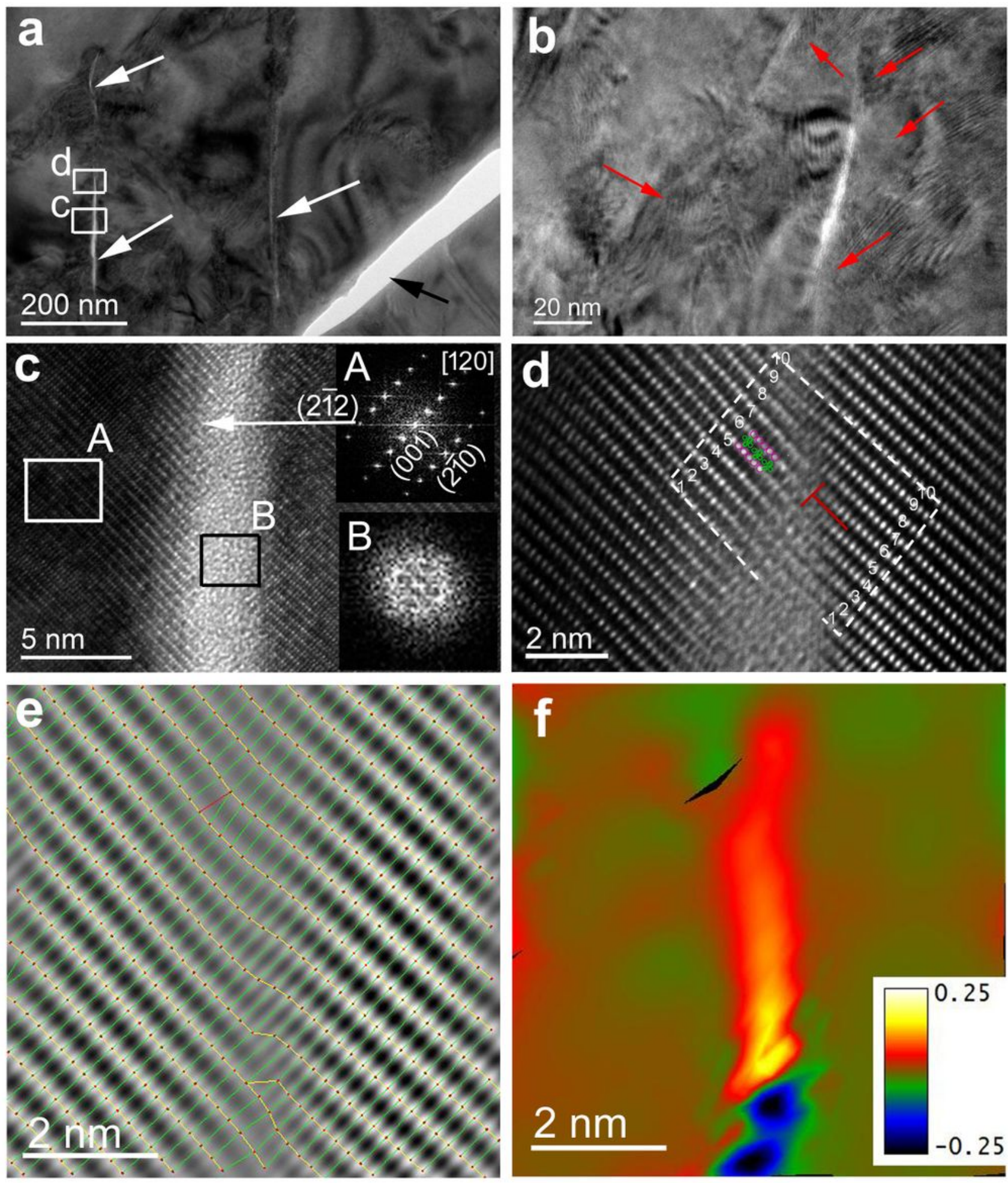

\section{Figure 3}

TEM observations underneath the indent region along [100] orientation of EuB6. a Low magnification bright field TEM image shows shear bands indicated by white arrows. b TEM image shows the planar defects at proximity of shear band. c High magnification TEM image shows the shear band along the (21 2) plane. The FFT of region A shows the crystalline diffraction spots along [120] zone axis while $B$ shows amorphous nature of diffuse halo ring. $d$ HRTEM image at the tip of the shear band shows emission of 
dislocations. The atomic overlay of Eu and B atoms on HRTEM shows a unit cell of EuB6 in which atomic colors are consistent with Fig. 1 (f). e Grid obtained by Peak Pair Analysis from the image shown in (d). $f$ shear strain (Exy) map obtained from same region of $(e)$.
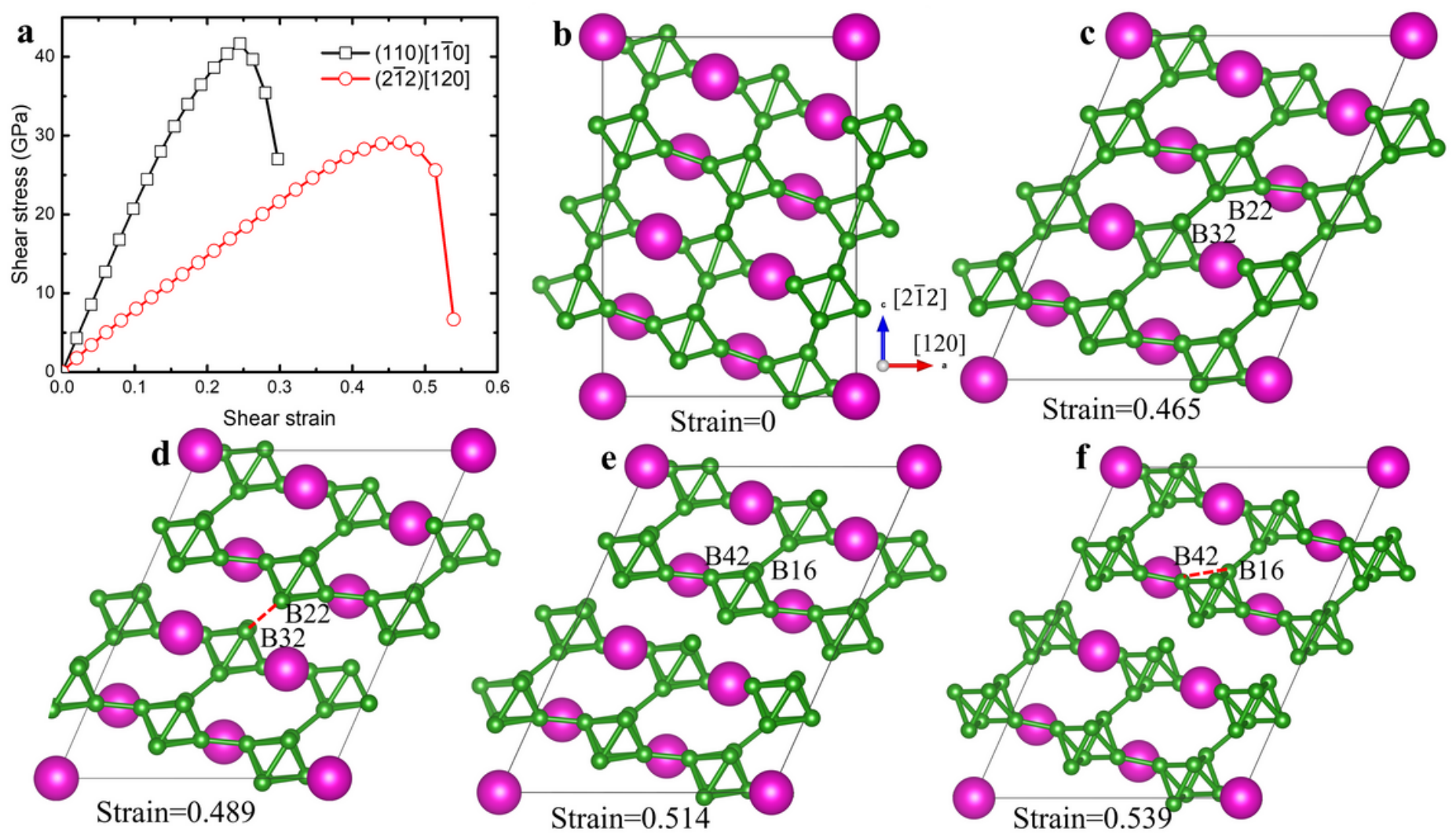

Figure 4

The deformation and failure process of EuB6 under pure shear deformation. a The shear-stress-shearstrain relationship along both $\left(2^{\prime \prime} 1^{\pi} 2\right)[120]$ and $(110)\left[1^{\prime \prime} 1^{\pi} 0\right]$ slip systems. (b-f) The failure process along $\left(2^{11} 1^{r} 2\right)[120]: b$ the intact structure; $c$ the structure at 0.465 strain before structural failure; $d$ the structure at 0.489 strain in which B22-B32 bond breaks; e the structure at 0.514 strain; $f$ the structure at 0.539 strain in which the B16-B42 bond breaks, initiating the deconstruction of octahedron. 

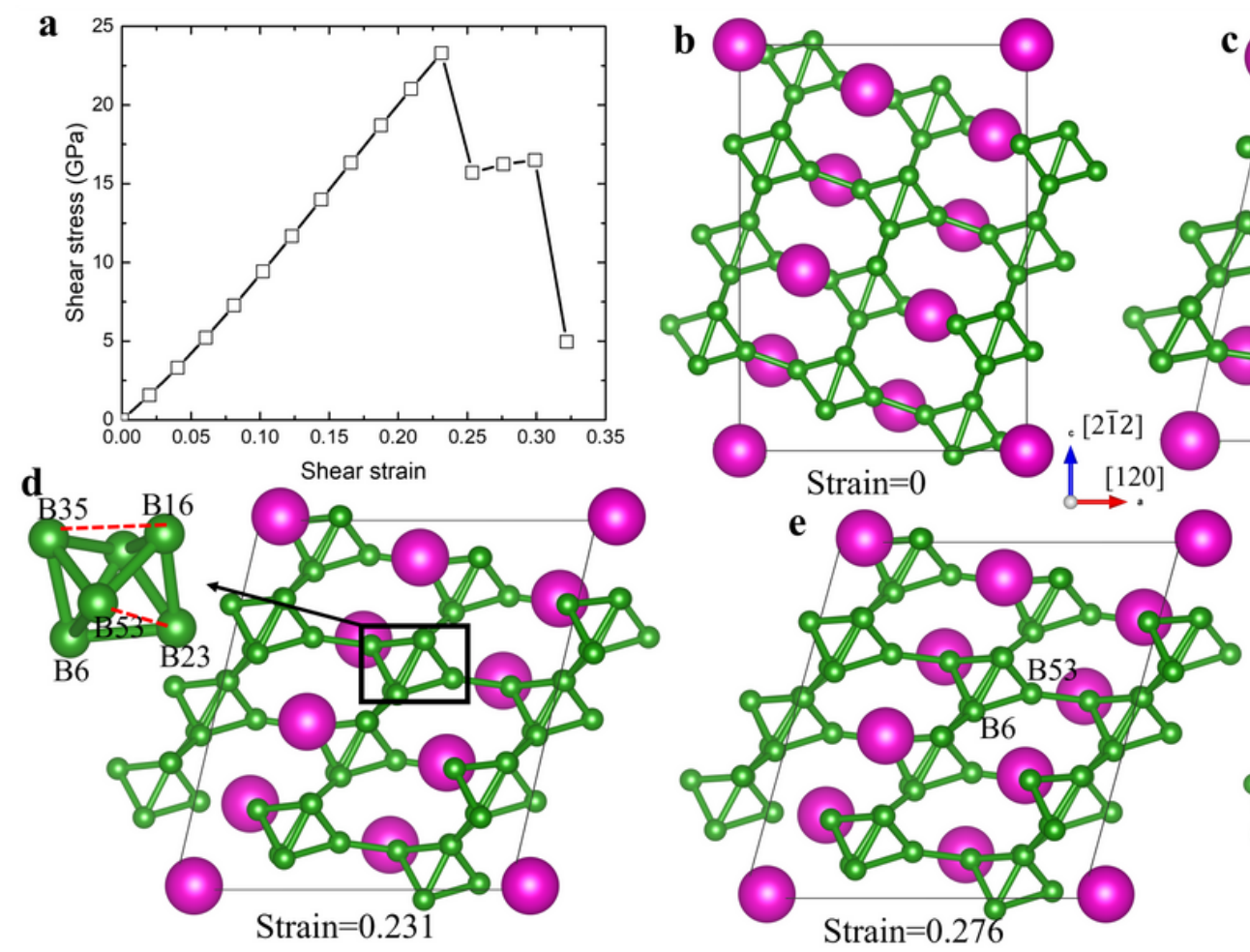

\section{Figure 5}

The failure process of EuB6 under biaxial shear deformation along ( $\left.2^{\prime \prime} 1^{\pi} 2\right)$ [120] slip system. a The shearstress-shear-strain relationship. (b-e) The failure process: $b$ the intact structure; $c$ the structure at 0.209 strain with ideal strength; $d$ the structure at 0.231 strain in which B35-B16 and B23-B53 bond within octahedron break; e the structure at 0.276 strain; $f$ the structure at 0.299 strain after structural failure. The B53-B6 bond within octahedron breaks.

\section{Supplementary Files}

This is a list of supplementary files associated with this preprint. Click to download.

- SupplementaryMaterialsNaturecommunications.docx 